\title{
Insurers' profits in the third-party liability insurance
}

\author{
Nico Dellaert and Hans Frenk \\ Erasmus University, 3000 DR Rotterdam, Netherlands

\section{Bob van der Laan \\ Goudse Verzekeringen, 2800 MA Gouda, Netherlands}

Received December 1989

Revised May 1991

\begin{abstract}
In this note we derive the expected total discounted profit of an insurer due to a single policy holder within a third-party liability insurance. We consider both a policy holder claiming optimally and non-optimally.
\end{abstract}

Keywords: Markov decision process, Optimal claim behaviour.

\section{Introduction}

We consider the Markov decision process as discussed in Dellaert et al. (1990) describing the optimal behaviour of a policy holder within an automobile third-party liability insurance governed by a bonus-malus rating system. For this model we derive a simple formula for the expected total discounted profit of the insurer due to this policy holder. Haehling von Lanzenauer (1972) discusses the same subject, but he uses the assumption that an individual causes damage at most once per year. Therefore his analysis is not suitable for practical situations. In the derivation of the result for a policy holder claiming optimally, we assume that the policy holder knows the exact duration of his insurance contract, his damage distribution and his damage rate. Because a lot of policy holders will not know this information, we will also present the results for a policy holder claiming reasonably. Finally we note that for a detailed analysis of the above decision model and the definition of the used state space the reader is referred to Dellaert et al. (1990). Also to simplify reading we will use the same notation as in Dellaert et al. (1990).

\section{Insurers' profit}

In this section we will determine the expected profit for the insurer due to a single policy holder. Clearly, profit due to a single policy holder is defined as the difference between the (discounted) costs and revenues over the entire insurance period. In the relevant literature very little attention has been paid to the concept of cost for the insurer. We can only refer to De Bakker (1987), Van den Bosch (1972) and Voute (1985). In practice one mostly reserves a percentage of the net premium for costs. We want to consider the costs for an insurer in more detail and therefore give a more detailed description of the components. Before starting this description we define a claim as an amount of money paid by the insurer to the policy holder for covering expenses caused by damages.

First, we have the fixed costs for each policy holder. These costs include for instance costs for administration of the policy contracts, overhead costs, costs made by other departments of the insurer in aid of the automobile insurance department, and advertising costs. Second, we have costs proportional to the policy holders net premium. Usually the main part is the commisssion for intermediaries. The third kind of costs are the fixed costs for cach claim, consisting mainly of costs for administration. The proportional costs for each claim are the last kind of costs. Apart from the claim size there are usually expert costs and additional administration costs which increase with the size of the claim.

Of course the gross premium for the insurance contains the expected costs for the above components and, additionally, reservations for bonusmalus discount and a profit component. The net premium is the amount the policy holder really pays (excluding taxes). This premium is determined by subtracting (or adding) the bonus (malus) discount from the gross premium. The bonus-malus discount is usually expressed as a 
percentage of the gross premium. An example of a bonus-malus discount system is presented in Table 1 of Dellaert et al. (1990). For the mathematical model considered the net premium is an exogenous variable. We remark that besides the costs there are, apart from the premium revenues, the interest revenues of premium and claim reserves. In this paper we neglect these revenues. They can, however, simply be taken into account by classifying them under one of the cost components.

\subsection{Analysis}

As in Dellaert et al. (1990) we assume that the policy holder is insured during a period of $T$ years. Each insurance year is divided into $N$ subperiods and at the end of each subperiod the policy holder has to decide whether or not to submit claims, if there have been damages during that period. The length of these subperiods is chosen in such a way that the probability of occurrence of two or more damages is negligible [an important difference with Haehling von Lanzenauer (1972)!], and hence either no damage or only one damage can occur in each subperiod. We will use the same state description as in Dellaert et al. (1990). The bonus-malus state $j$ is determined by two elements:

1. the premium to be paid next year by the policy holder when he does not claim during the rest of the current year $\left(\pi_{0}\right)$,

2. the premium to be paid next year by the policy holder when he claims exactly one damage during the rest of the current year $\left(\pi_{1}\right)$.

We can elicit every possible combination of $\pi_{0}$ and $\pi_{1}$ from the transition table of the bonusmalus scheme [cf. Dellaert et al. (1990, Table 1)]. We let $j$ denote a particular combination, where $j=1, \ldots, J$. The combination $\left(\pi_{0}, \pi_{1}\right)$ does not change if there is no claim. We now consider two cases where $j$ changes.

1. The policy holder claims a damage. After a claim the policy holder moves to a new combination $a(j)=\left(\pi_{0}^{*}, \pi_{1}^{*}\right)$, where $\pi_{0}^{*}=\pi_{1}$. It follows that is not necessary to record the number of claims during the insurance year, because next year's premium is adapted immediately after a claim.
Table 1

Bonus-malus system.

\begin{tabular}{lrrrrr}
\hline$j$-value & $\pi_{0}(j)$ & $\pi_{1}(j)$ & $u(j)$ & $b(j)$ & BM-class \\
\hline 1 & 1200 & 1200 & 1 & 2 & - \\
2 & 1000 & 1200 & 1 & 3 & 1 \\
3 & 900 & 1200 & 1 & 4 & 2 \\
4 & 800 & 1200 & 1 & 5 & 3 \\
5 & 700 & 1200 & 1 & 7 & 4 \\
6 & 600 & 1200 & 1 & 9 & - \\
7 & 600 & 1000 & 2 & 9 & 5 \\
8 & 550 & 1000 & 2 & 10 & \\
9 & 550 & 900 & 3 & 10 & 6 \\
10 & 500 & 800 & 4 & 11 & 7 \\
11 & 450 & 700 & 5 & 12 & 8 \\
12 & 400 & 600 & 6 & 13 & 9 \\
13 & 375 & 550 & 8 & 14 & 10 \\
14 & 350 & 550 & 9 & 15 & 11 \\
15 & 325 & 500 & 10 & 16 & 12 \\
16 & 300 & 500 & 10 & 17 & 13 \\
17 & 300 & 450 & 11 & 17 & 14 \\
\hline
\end{tabular}

2. A new insurance year starts. The policy holder pays the premium at the beginning of each insurance year. This premium is equal to $\pi_{0}(a(j))$ or $\pi_{0}(j)$ depending on whether he has claimed during the last period of the previous insurance year. When he does not claim during the new insurance year he will move to a higher bonus-malus class next year (unless he is in the highest class) and pay a lower premium. This corresponds to a different combination $\left(\pi_{0}, \pi_{1}\right)$. We will describe the number of the new combination by $b(j)$ if there has been no claim during the last period of the previous year and by $b(a(j))$ if there has been one claim.

An example of this bonus-malus system with 14 bonus-malus classes [based on Table 1 of Dellaert et al. (1990), with a gross premium of Dfl. 1000] is presented in Table 1. In this table we find the number of the bonus-malus combinations $j$, the next-year premium (with and without claiming), the transitions and the corresponding bonus-malus class (BM-class) at the beginning of the insurance year. Note that the bonus-malus combinations 1,6 and 8 can only be reached after there has been a claim during the insurance year and so they cannot occur at the beginning of the year.

In order to determine the expected total discounted profit of the insurer we introduce the following functions. Let $i$ be the number of remaining years (or a part of the year) of the 
insurance, $1 \leq i \leq T$, let $n$ be the subperiod in the current year, $1 \leq n \leq N$ and let $j$ be the bonusmalus combination, $1 \leq j \leq J$. The costs or profit from the end of period $N(T-i)+n$ through the end of period $N T$ for an optimally claiming policy holder at the end of period $N(T-i)+n$, who is in bonus-malus combination $j$ just before submitting possible claims are defined by:

$S_{i 1}(j, n)=$ expected total discounted costs of the insurer due to claimed damages and the proportional extra costs;

$S_{i 2}(j, n)=$ expected total discounted costs due to damages paid by an optimally claiming policy holder himself;

$S_{i}(n) \quad=$ expected total discounted damages faced by a policy holder;

$W_{i}(j, n)=$ expected total discounted profit of the insurer;

$L_{i}(j, n)=$ expected total discounted costs of the insurer due to fixed costs of claims.

Moreover, let

$W_{T}(j)=$ expected total discounted profit of the insurer from the beginning of period 1 through the end of period NT due to an optimally claiming policy holder, who just entered the insurance in bonus-malus combination $j$.

By the above definitions, it follows immediately that

$W_{T}(j)=R(j)+\beta W_{T}(j, 1)$,

where $R(j)$ denotes the revenue of the insurer at the beginning of period 1 due to the entrance of a policy holder in bonus-malus combination $j$ and $\beta<1$ is the one-subperiod discount factor. Clearly, if the random variable $\boldsymbol{X}_{k}$ denotes the total amount of damage in subperiod $k, 1 \leq k \leq$ $N T$, it is easy to give a simple formula for $S_{i}(n)$ :

$$
\begin{aligned}
S_{i}(n) & =\mathbb{E}\left(\sum_{k=N(T-i)+n}^{N T} \beta^{k-N(T-i)-n} \boldsymbol{X}_{k}\right) \\
& =\sum_{k=N(T-i)+n}^{N T} \beta^{k-N(T-i)-n} \mathbb{E}\left(\boldsymbol{X}_{k}\right)
\end{aligned}
$$

with $\mathbb{E}$ denoting mathematical expectation, and so $S_{i}(n)$ is easy to calculate for every $1 \leq i \leq T$ and $1 \leq n \leq N$.

For reasons of convenience we will assume that the damage $\boldsymbol{X}_{k}$ is independent of the subpe- riod $k$ and can simply be described by $\boldsymbol{X}$. This implies that, if the expected damage during a subperiod equals a constant $s$, we have

$S_{i}(n)=\frac{1-\beta^{N i-n+1}}{1-\beta} s$.

We will assume that the extra proportional costs for the insurer due to claims are equal to a fraction $\gamma$ of the claimed damage. Since damages either have to be paid by the insurer or by the policy holder the next equality follows by definition:

$S_{i}(n)=S_{i 1}(j, n) /(1+\gamma)+S_{i 2}(j, n)$

for every $1 \leq i \leq T, 1 \leq n \leq N$ and $1 \leq j \leq J$. Hence for the computation of $S_{i 1}(j, n)$ and $S_{i 2}(j, n)$ it is sufficient by (2) and (4) to compute only $S_{i 1}(j, n)$. This computation, as will be shown, can be carried out recursively once we know the optimal claim policy. This policy can be obtained as follows. Let [cf. Dellaert et al. (1990)]

$\begin{aligned} V_{i}(j, n, x)= & \text { minimal expected total discounted } \\ & \text { costs faced by a policy holder from } \\ & \text { the end of period } N(T-i)+n \\ & \text { through the end of period } N T \text { if } \\ & \text { this policy holder is in bonus-malus } \\ & \text { combination } j \text { and faces a damage } \\ & \text { of size } x \text { at the end of period } N(T \\ & -i)+n\end{aligned}$ for every $1 \leq i \leq T, 1 \leq n \leq N, 1 \leq j \leq J$ and $x \geq 0$ and define

$V_{i}(j, n)=\mathbb{E}\left(V_{i}(j, n, X)\right)$.

In Dellaert et al. (1990) it is proved that the optimal claim policy for a finite horizon model with $N T$ periods is a so-called threshold policy. The threshold policy has the following form: claim a particular damage $x$ at the end of period $N(T$ $-i)+n$ observing combination $j$ if and only if $x>D_{i}(j, n)$ where

$$
D_{i}(j, n)=\beta\left(V_{i}(a(j), n+1)-V_{i}(j, n+1)\right)
$$

whenever $1 \leq i \leq T, 1 \leq n \leq N-1,1 \leq j \leq J$ and

$$
\begin{aligned}
D_{i}(j, N)= & \beta\left(V_{i-1}(b(a(j)), 1)\right. \\
& \left.-V_{i-1}(b(j), 1)\right)+\pi_{0}(a(j)) \\
& -\pi_{0}(j)
\end{aligned}
$$

whenever $2 \leq i \leq T, 1 \leq j \leq J$. Moreover, it is also proved in Dellaert et al. (1990) that the complex- 
ity of the algorithm to compute the threshold values $D_{i}(j, n)$ is $O(N J T)$. By the above observations it is not difficult to compute $S_{i 1}(j, n)$ and $L_{i}(j, n)$ recursively. In order to write out this recursive scheme, we define

$\alpha \quad=$ fixed costs for each claim which leads to indemnity;

$c_{i}(j, n)=$ expected number of claims which lead to indemnity in period $N(T-i)+n$ of an optimaly claiming policy holder, if this policy holder, just before submitting possible claims, is in bonus-malus combination $j$ at the end of period $N(T-i)+n$;

$d_{i}(j, n)=$ expected amount of claimed damage due to an optimally claiming policy holder in period $N(T-i)+n$, if this policy holder, just before submitting possible claims, is in bonus-malus combination $j$ at the end of period $N(T-i)+n$.

By the form of the optimal claiming policy and the occurrence of at most one damage in each subperiod we obtain

$c_{i}(j, n)-1-G\left(D_{i}(j, n)\right)$

with $G(d):=\mathbb{P}\{X \leq d\}$ and

$d_{i}(j, n)=\mathbb{E} \boldsymbol{X}-K\left(D_{i}(j, n)\right)$

with $K(d):=\mathbb{E}\left(X 1_{\{X \leq d\}}\right)$. Hence $c_{i}(j, n)$ also equals the probability of a claim in period $N(T-$ $i)+n$ and so we have for any $1 \leq i \leq T$ and $1 \leq j \leq J$ :

$L_{i}(j, n)=\left\{\begin{array}{l}\alpha c_{i}(j, n)+\beta\left\{\left(1-c_{i}(j, n)\right) L_{i}(j, n+1)\right. \\ \left.\quad+c_{i}(j, n) L_{i}(a(j), n+1)\right\} \\ \text { if } 1 \leq n \leq N-1, \\ \alpha c_{i}(j, N)+\beta\left\{\left(1-c_{i}(j, N)\right) L_{i-1}(b(j), 1)\right. \\ \left.\quad+c_{i}(j, N) L_{i-1}(b(a(j)), 1)\right\} \\ \text { if } n=N .\end{array}\right.$

Moreover, by a similar standard argument

$$
S_{i 1}(j, n)=\left\{\begin{array}{c}
(1+\gamma) d_{i}(j, n) \\
\quad+\beta\left\{\left(1-c_{i}(j, n)\right) S_{i 1}(j, n+1)\right. \\
\left.\quad+c_{i}(j, n) S_{i 1}(a(j), n+1)\right\} \\
\text { if } 1 \leq n \leq N-1, \\
(1+\gamma) d_{i}(j, N) \\
\quad+\beta\left\{\left(1-c_{i}(j, N)\right) S_{i-1,1}(b(j), 1)\right. \\
\left.\quad+c_{i}(j, N) S_{i-1,1}(b(a(j)), 1)\right\} \\
\text { if } n=N .
\end{array}\right.
$$

Observe that the above formulae simplify in the last year of the insurance, since in this last year the policy holder will always claim, i.e. $D_{T}(j, n)$ $=0$ for every $1 \leq n \leq N$. The recursive procedures in (10) and (11) take care of the computation of the cost components related to damages. We still have to analyse the cost components related to the insurance policy before stating our main theorem. Therefore we define:

$\delta \quad=$ fraction of the net premium to be paid to the intermediary;

$C \quad=$ fixed costs per year for each policy holder; $F_{i}(n)=$ discounted fixed costs per policy holder from the end of period $N(T-i)+n$ through the end of period $N T$.

For convenience, we assume that the fixed costs per policy holder and the intermediary's commission will be paid at the beginning of each insurance year. Due to this assumption we have for $1 \leq n \leq N$,

$F_{i}(n)= \begin{cases}0 & \text { if } i=1, \\ C \beta^{N-n+1} \sum_{k=0}^{i-2} \beta^{N k} & \text { if } 2 \leq i \leq T .\end{cases}$

Finally, we introduce

$V_{T}(j)=$ expected total discounted costs faced by an optimally claiming policy holder from the beginning of period 1 through the end of period $N T$, if this policy holder enters the insurance in bonus-malus combination $j$.

Almost by definition the next result holds.

Theorem 2.1. For any $1 \leq i \leq T, 1 \leq n \leq N$ and $1 \leq j \leq J$ we have

$$
\begin{aligned}
W_{i}(j, n)= & (1-\delta)\left[V_{i}(j, n)-S_{i}(n)\right] \\
& -(\delta+\gamma) S_{i 1}(j, n) /(1+\gamma) \\
& -L_{i}(j, n)-F_{i}(n) .
\end{aligned}
$$

Moreover, if $\pi(j)$ denotes the premium to be paid to enter the bonus-malus class combination $j$ at the start of the insurance, we obtain

$W_{T}(j)=(1-\delta) \pi(j)-C+\beta W_{T}(j, 1)$.

Proof. Let [cf. Dellaert et al. (1990)]

$P_{i}(j, n, x)=$ expected discounted premiums received by the insurer from the end 
of period $N(T-i)+n$ through the end of period $N T$ due to an optimally claiming policy holder, if this policy holder is in bonus-malus combination $j$ and faces a damage of size $x$ at the end of period $N(T$ $-i)+n$

for any $1 \leq i \leq T, 1 \leq n \leq N, 1 \leq j \leq J$ and $x \geq 0$ and define

$P_{i}(j, n):=\mathbb{E} P_{i}(j, n, \boldsymbol{X})$.

Clearly by definition of the cost components, we obtain

$$
\begin{aligned}
W_{i}(j, n)= & (1-\delta) P_{i}(j, n)-S_{i 1}(j, n) \\
& -L_{i}(j, n)-F_{i}(n) .
\end{aligned}
$$

Moreover [cf. Dellaert et al. (1990)],

$V_{i}(j, n)=P_{i}(j, n)+S_{i 2}(j, n)$

and hence by (4), (12) and (13) the first part follows. For the proof of the second part we note that $R(j)$ equals $(1-\delta) \pi(j)-C$ and hence by (1) the desired result is obtained.

This proof concludes this section. In the next section we will discuss non-optimal claim sizes and investigate their influence on the profit expectations.

\section{Non-optimal behaviour of the policy holder}

If the policy holder uses non-optimal claim sizes, his expected total discounted costs will obviously be larger than the minimal expected total discounted costs. Due to Theorem 2.1 the expected total discounted profit for the insurer will change due to this difference. It is interesting to consider the difference in expected total discounted profit between a policy holder who uses optimal claim sizes and a policy holder using reasonable, but non-optimal claim sizes. We believe that claim sizes based on the following assumptions are reasonable:

1. The policy holder believes that he will have no more damages during the remaining part of the insurance contract.

2. During the last year of the insurance contract (year $T$ ) the policy holder knows that the insurance will be ended by the end of that year. During earlier years the policy holder assumes to be insured 'forever'.

3. The policy holder used a discount rate $\beta=1$.

The claim sizes following from these assumptions can be calculated easily. During the last year of the insurance (year $T$ ) all damages will be claimed and this corresponds with

$D_{1}(j, n)=0$

for all $1 \leq n \leq N$ and $1 \leq j \leq J$. During earlier years we consider the difference in the premium that has to be paid if damage is claimed or if no damage is claimed. We consider therefore two sequences, $K_{1}(j), \quad K_{2}(j), \ldots$ and $M_{1}(j), M_{2}$ $(j), \ldots$, where $K_{l}(j)$ is the bonus-malus combination of the policy holder $l$ years from now if the claims in the present year and $M_{l}(j)$ the bonus-malus combination if he does not claim in the prcsent year while he is in bonus-malus combination $j$. Due to Assumption 1 we have the following formulae:

$$
\begin{array}{ll}
K_{l}(j)=a(j) & \text { if } l=1, \\
K_{l}(j)=b\left(K_{l-1}(j)\right) & \text { if } l \geq 2,
\end{array}
$$

and

$$
\begin{array}{ll}
M_{l}(j)=j & \text { if } l=1, \\
M_{l}(j)-b\left(M_{l-1}(j)\right) & \text { if } l \geq 2 .
\end{array}
$$

After a number of years the claiming policy holder will enter the combination $J$ with the smallest amount of premium. From that year onwards there are no more differences in premium between the two options. Now let

$L(j)=\min \left\{l \mid K_{l}(j)=J\right\}$.

Then the claim size threshold is determined by the differences in premium during the first $L(j)$ -1 years:

$D_{i}(j, n)=\sum_{l=1}^{L(j)}\left(\pi_{0}\left(K_{l}(j)\right)-\pi_{0}\left(M_{l}(j)\right)\right)$

for $1 \leq n \leq N, 1 \leq j \leq J$ and $1 \leq i<T$.

One other interesting behaviour of a policy holder is the one in which the policy holder uses only one positive claim size, next to the obvious claim size treshold 0 for bonus-malus combination 1 and during the last year of the insurance. There is not a unique best value for this claim size which is independent from the bonus-malus 
combination or the duration of the insurance. In the next section we consider a rather arbitrary value.

\section{Numerical results}

In this section we calculate the expected total discounted profit for the example given in Dellaert et al. (1990). In this example the amount of damage is lognormally distributed, with an average of Dfl. 1800, the basic premium is Dfl. 1000, the fixed costs $C$ for each policy holder are Dfl. 75 , the fixed costs $\alpha$ per claim are Dfl. 100, the fraction $\gamma$ of proportional costs per claim equals 0.1 and the fraction $\delta$ to be paid to the intermediary is 0.2 . We take $N=12$, the number of subperiods in a year, and a discount rate per year equal to 0.95 , so $\beta=0.99594$. We consider different damage rates.

First we consider the differences between the rcasonable claim size and the optimal claim size policies. For further information on the behaviour of the optimal claim sizes we refer to Dellaert et al. (1990). We remark that the values of the reasonable claim sizes do not depend on the damage rate. In Table 2 we compare the values with the optimal claim sizes, for which we consider two different damage rates: 0.1 and 0.2 . These values are based on a stationary situation with an infinite horizon. The optimal claim sizes are different for every subperiod $n$ in an insurance year. The values presented in Table 2 are
Table 2

Reasonable and optimal claim sizes.

\begin{tabular}{rrcc}
\hline$j$ & Rcasonable & $\begin{array}{l}\text { Optimal } \\
\text { (rate 0.1) }\end{array}$ & $\begin{array}{c}\text { Optimal } \\
\text { (rate 0.2) }\end{array}$ \\
\hline 1 & 0 & 0 & 0 \\
2 & 900 & 711 & 662 \\
3 & 1600 & 1299 & 1243 \\
4 & 2200 & 1840 & 1803 \\
5 & 2700 & 2320 & 2324 \\
6 & 3100 & 2728 & 2764 \\
7 & 2200 & 2012 & 2115 \\
8 & 2500 & 2326 & 2462 \\
9 & 1800 & 1737 & 1894 \\
10 & 1450 & 1465 & 1647 \\
11 & 1150 & 1205 & 1385 \\
12 & 900 & 977 & 1146 \\
13 & 700 & 778 & 921 \\
14 & 775 & 862 & 1020 \\
15 & 575 & 659 & 790 \\
16 & 600 & 697 & 842 \\
17 & 400 & 483 & 598 \\
\hline
\end{tabular}

constructed by taking the average over all subperiods. [cf. Table 5.4 of Dellaert et al. (1990).]

In Table 2 we find the differences between the reasonable claim sizes and the averaged optimal claim sizes to be rather small. We have also found small differences between the reasonable claim sizes and the optimal claim sizes during the first years of a finite horizon situation. From the small differences we expect that the expected total discounted profit for the reasonable claim sizes will not differ much from the profit with the

Table 3

Comparison between profits.

\begin{tabular}{|c|c|c|c|c|c|c|}
\hline \multirow{2}{*}{$\begin{array}{l}\text { BM-class } \\
\text { at entry }\end{array}$} & \multicolumn{3}{|l|}{ Rate 0.2} & \multicolumn{3}{|l|}{ Rate 0.1} \\
\hline & Optimal & Reasonable & Fixed & Optimal & Reasonable & $\overline{\text { Fixed }}$ \\
\hline 1 & 1585 & 1651 & 1650 & 3432 & 3458 & 3489 \\
\hline 2 & 1020 & 1073 & 1073 & 2831 & 2853 & 2887 \\
\hline 3 & 522 & 560 & 585 & 2333 & 2349 & 2397 \\
\hline 4 & 33 & 55 & 109 & 1870 & 1881 & 1944 \\
\hline 5 & -432 & -424 & -354 & 1452 & 1458 & 1531 \\
\hline 6 & -827 & -826 & -743 & 1107 & 1109 & 1193 \\
\hline 7 & -1146 & -1148 & -1052 & 837 & 838 & 934 \\
\hline 8 & -1422 & -1426 & -1316 & 607 & 607 & 716 \\
\hline 9 & -1648 & -1653 & -1527 & 420 & 419 & 542 \\
\hline 10 & -1820 & -1825 & -1686 & 279 & 278 & 410 \\
\hline 11 & -1932 & -1937 & -1789 & 186 & 184 & 324 \\
\hline 12 & -2041 & -2045 & -1883 & 103 & 102 & 251 \\
\hline 13 & -2105 & -2109 & -1938 & 52 & 51 & 205 \\
\hline 14 & -2158 & -2162 & -1980 & 17 & 15 & 176 \\
\hline
\end{tabular}


optimal claim-sizes. We also consider the profit for the policy holder using only one claim size, in this case Dfl. 1000. In Table 3 we present the expected discounted profit for a policy holder using the optimal claim-sizes, one using the reasonable claim sizes and one using a fixed claim size. The profit is calculated for two different damage rates. The policy holder enters the insurance for a period of 25 years in one of the 14 bonus-malus classes.

Some important conclusions can be drawn form Table 3. The most important element is of course the surprisingly small difference between the expected discounted profit for a policy holder using optimal claim sizes and a policy holder using reasonable claim sizes. In this table we find that for a damage rate of 0.1 the difference is always less than Dfl. 26. For a damage rate of 0.2 the difference does not exceed Dfl. 66. For BMclasses 5 and up the differences are almost negligible. (We find the same small differences when we consider the expected discounted costs for the policy holder!)

Using only one claim size leads to much larger costs for the policy holder and also to a larger profit for the insurer. The differences between this policy and the optimal policy is more or less independent from the damage rate.

In order to give an indication of the course of the profit size, we will present two tables in which we consider different lengths of the insurance period. In Table 4 we consider five different insurance periods and a damage rate of 0.1 . In

Table 4

Profit for different insurance periods.

\begin{tabular}{lrrrrl}
\hline BM-class & \multicolumn{1}{l}{5} & \multicolumn{1}{l}{10} & \multicolumn{1}{l}{15} & \multicolumn{1}{l}{20} & \multicolumn{1}{l}{25} \\
\hline 1 & 2288 & 3103 & 3341 & 3406 & 3432 \\
2 & 1890 & 2567 & 2753 & 2807 & 3831 \\
3 & 1580 & 2124 & 2265 & 2310 & 2333 \\
4 & 1283 & 1703 & 1809 & 1848 & 1870 \\
5 & 1002 & 1313 & 1397 & 1431 & 1452 \\
6 & 758 & 988 & 1055 & 1086 & 1107 \\
7 & 571 & 734 & 788 & 817 & 837 \\
8 & 406 & 516 & 559 & 587 & 607 \\
9 & 263 & 336 & 374 & 400 & 420 \\
10 & 146 & 198 & 233 & 259 & 279 \\
11 & 67 & 107 & 140 & 166 & 186 \\
12 & -3 & 27 & 58 & 83 & 103 \\
13 & -49 & -23 & 7 & 32 & 52 \\
14 & -80 & -58 & -28 & -3 & 17 \\
\hline
\end{tabular}

Table 5

Profit for different insurance periods.

\begin{tabular}{lrrrrr}
\hline BM-class & \multicolumn{1}{c}{5} & \multicolumn{1}{c}{10} & \multicolumn{1}{c}{15} & \multicolumn{1}{c}{20} & \multicolumn{1}{c}{25} \\
\hline 1 & 1337 & 1924 & 1914 & 1756 & 1585 \\
2 & 985 & 1446 & 1376 & 1196 & 1020 \\
3 & 706 & 1031 & 902 & 704 & 522 \\
4 & 420 & 612 & 430 & 218 & 33 \\
5 & 131 & 199 & -22 & -245 & -432 \\
6 & -124 & -153 & -407 & -638 & -827 \\
7 & -326 & -439 & -718 & -955 & -1146 \\
8 & -508 & -691 & -988 & -1230 & -1422 \\
9 & -664 & -900 & -1211 & -1456 & -1648 \\
10 & -790 & -1061 & -1380 & -1627 & -1820 \\
11 & -873 & -1167 & -1491 & -1739 & -1932 \\
12 & -958 & -1270 & -1599 & -1848 & -2041 \\
13 & -1010 & -1331 & -1662 & -1912 & -2105 \\
14 & -1054 & -1382 & -1715 & -1965 & -2158 \\
\hline
\end{tabular}

Table 5 we consider the same insurance periods for a damage rate of 0.2 .

From Table 4 we can learn that the differences between an insurance period of 10 years and one of 25 years are quite small. This implies that the outcome is relatively insensitive for the exact length of the insurance period and also that the size of the premium is quite attractive from the insurers point of view. The results of Table 5 are quite different. Here, the expected discounted profit over a period of 25 years is negative for the majority of the BM-classes. This indicates that it will not be very profitable to admit policy holders with an expected damage rate of 0.2 damages per year in BM-class 5 or higher. Admittance in one

Table 6

Undiscounted profit for different insurance periods.

\begin{tabular}{lrrrrr}
\hline BM-class & \multicolumn{1}{l}{5} & \multicolumn{1}{c}{10} & \multicolumn{1}{c}{15} & \multicolumn{1}{c}{20} & \multicolumn{1}{c}{25} \\
\hline 1 & 1545 & 2053 & 1910 & 1418 & 806 \\
2 & 1165 & 1495 & 1240 & 705 & 80 \\
3 & 858 & 1000 & 641 & 69 & -565 \\
4 & 547 & 505 & 58 & -540 & -1181 \\
5 & 236 & 23 & -487 & -1104 & -1750 \\
6 & -40 & -388 & -948 & -1579 & -2229 \\
7 & -260 & -721 & -1320 & -1961 & -2613 \\
8 & -455 & -1010 & -1637 & -2286 & -2940 \\
9 & -622 & -1248 & -1895 & -2548 & -3203 \\
10 & -757 & -1430 & -2089 & -2746 & -3402 \\
11 & -847 & -1550 & -2217 & -2875 & -3532 \\
12 & -938 & -1665 & -2337 & -2997 & -3654 \\
13 & -993 & -1731 & -2407 & -3068 & -3725 \\
14 & -1038 & -1786 & -2464 & -3125 & -3782 \\
\hline
\end{tabular}


of these classes should be combined with a higher premium.

This becomes even more clear if we consider the results of the undiscounted situation with damage rate 0.2 , which is presented in Table 6 . In this table we notice that for an insurance period of 25 years only admittance in one of the first two $\mathrm{BM}$-classes is profitable for the insurer.

\section{References}

De Bakker, A.C.M. (1987). Kostenproblemen bij verzekeringsmaatschappijen. In: Economie van het Verzekeringsbedrijf. Kluwer Bedrijswetenschappen, Deventer.

Dellaert, N.P., J.B.G. Frenk, A. Kouwenhoven and B.S. van der Laan (1990). Optimal claim behaviour for third-party liability insurances or to claim or not to claim - that is the question. Insurance: Mathematics and Economics 9, 59-76.

Haehling von Lanzenauer, C. (1972). The effect of the insurees decisions on the insurers profit. Proceedings in Operations Research, 399-421.

Van den Bosch, P.J.H.J. (1952). Het kostenprobleem in de levensverzekering. Verzekerings-Archief, Vol. XXX, 1-31.

Voute, A.B.E. (1985). Op zoek naar een kostprijs van verzekeringen. Inaugural speech. Rotterdam. 